\title{
(6) OPEN ACCESS \\ EULAR recommendations for women's health and the management of family planning, assisted reproduction, pregnancy and menopause in patients with systemic lupus erythematosus and/or antiphospholipid syndrome
}

\author{
L Andreoli, ${ }^{1,2}$ G K Bertsias, ${ }^{3}$ N Agmon-Levin, ${ }^{4,5}$ S Brown, ${ }^{6}$ R Cervera, ${ }_{1}^{7}$ \\ $\mathrm{N}$ Costedoat-Chalumeau, ${ }^{8,9}$ A Doria, ${ }^{10}$ R Fischer-Betz, ${ }^{11} \mathrm{~F}$ Forger, ${ }_{1}^{12}$ \\ M F Moraes-Fontes ${ }^{13} \mathrm{M}$ Khamashta, ${ }^{14,15} \mathrm{~J}$ King, ${ }^{16}$ A Lojacono ${ }^{1,17} \mathrm{~F}$ Marchiori, ${ }^{18}$ \\ $\mathrm{P}$ L Meroni, ${ }^{19} \mathrm{M}$ Mosca, ${ }^{20} \mathrm{M}$ Motta, ${ }^{21} \mathrm{M}$ Ostensen, ${ }^{22} \mathrm{C}$ Pamfil ${ }^{23} \mathrm{~L} \mathrm{Raio}^{24}$ \\ M Schneider, ${ }^{11}$ E Svenungsson, ${ }^{25} \mathrm{M}$ Tektonidou, ${ }^{26} \mathrm{~S}$ Yavuz, ${ }^{27}$ D Boumpas, ${ }^{28,29}$ \\ A Tincani ${ }^{1,2}$
}

Handling editor Tore K Kvien

- Additional material is published online only. To view please visit the journal online (http://dx.doi.org/10.1136/ annrheumdis-2016-209770)

For numbered affiliations see end of article.

Correspondence to

Professor Angela Tincani, Unit of Rheumatology and Clinical Immunology, ASST Spedali Civili, Piazzale Spedali Civili, 1, Brescia 25123, Italy; angela.tincani@unibs.it

LA and GKB contributed equally;

DB and AT share senior authorship.

Received 22 April 2016 Revised 13 June 2016 Accepted 25 June 2016 Published Online First 25 July 2016

\section{ABSTRACT}

Objectives Develop recommendations for women's health issues and family planning in systemic lupus erythematosus (SLE) and/or antiphospholipid syndrome (APS).

Methods Systematic review of evidence followed by modified Delphi method to compile questions, elicit expert opinions and reach consensus.

Results Family planning should be discussed as early as possible after diagnosis. Most women can have successful pregnancies and measures can be taken to reduce the risks of adverse maternal or fetal outcomes. Risk stratification includes disease activity, autoantibody profile, previous vascular and pregnancy morbidity, hypertension and the use of drugs (emphasis on benefits from hydroxychloroquine and antiplatelets/anticoagulants). Hormonal contraception and menopause replacement therapy can be used in patients with stable/inactive disease and low risk of thrombosis. Fertility preservation with gonadotropin-releasing hormone analogues should be considered prior to the use of alkylating agents. Assisted reproduction techniques can be safely used in patients with stable/inactive disease; patients with positive antiphospholipid antibodies/APS should receive anticoagulation and/or low-dose aspirin. Assessment of disease activity, renal function and serological markers is important for diagnosing disease flares and monitoring for obstetrical adverse outcomes. Fetal monitoring includes Doppler ultrasonography and fetal biometry, particularly in the third trimester, to screen for placental insufficiency and small for gestational age fetuses. Screening for gynaecological malignancies is similar to the general population, with increased vigilance for cervical premalignant lesions if exposed to immunosuppressive drugs. Human papillomavirus immunisation can be used in women with stable/inactive disease.

Conclusions Recommendations for women's health issues in SLE and/or APS were developed using an evidence-based approach followed by expert consensus.

\section{INTRODUCTION}

Systemic lupus erythematosus (SLE) and the antiphospholipid syndrome (APS), SLE-associated or primary APS, affect mostly women of childbearing age. Several 'unmet needs' in the management of reproductive and other women's health issues may impact on personal relationships and the decision to have children. ${ }^{1}$ Because of earlier recognition of disease and advances in medical treatment, family planning has gained greater importance. ${ }^{2-4}$ Concerns include the effect of pregnancy on maternal disease, the impact of disease activity on fetal health and the safety of medications during pregnancy and breast feeding. Assessment of fertility and feasibility of assisted reproduction techniques (ARTs), use of contraception, management of menopause and surveillance against malignancies need to be addressed. We gathered a multidisciplinary panel of experts to develop evidencebased recommendations on the management of family planning and women's health issues in SLE and/or APS.

\section{METHODS}

We followed the European League Against Rheumatism (EULAR) standardised operating procedures $^{5}$ and the Appraisal of Guidelines Research and Evaluation instrument. Through a Delphibased approach, the committee selected 12 research questions further edited for systematic literature review (see online supplementary table S1). We searched PubMed using arrays of relevant terms; all English-language publications up to December 2014 were considered. A hand search was also performed in October 2015. Retrieved items were refined based on abstract, full-text content and number of included patients. A detailed presentation of the literature review is given in the online supplementary table S2. Evidence was categorised based on the design and validity of available studies and the strength of the statements was graded (see online supplementary table S3). After rounds of 
Table 1 Recommendations for women's health and the management of family planning, assisted reproduction, pregnancy and menopause in patients with systemic lupus erythematosus (SLE) and/or antiphospholipid syndrome (APS)

LoA

\section{Statement/recommendation}

1. Preconception counselling and risk stratification

1.1 In women with SLE, major risk factors for adverse maternal and fetal outcomes include active/flaring SLE (1/A), especially active nephritis (1/A), history of lupus nephritis (2/B) and presence of aPL/APS ${ }^{*}(1 / A)$

1.1.1 Blood pressure monitoring (2/B), use of safe medications to control disease activity (emphasis on HCQ (2/B)) and limiting glucocorticoids exposure (2/B) are essential measures.

1.2 In women with APS (primary or SLE-APS), risk factors include high-risk aPL profile (lupus anticoagulant, multiple aPL, moderate to high titre aPL) (1/A), coexisting SLE (2/B), history of vascular/thrombotic APS (2/B) and of previous adverse pregnancy complications (2/B).

1.2.1 Blood pressure monitoring ( $3 / C$ ) and use of antiplatelet and/or anticoagulant therapy (rated at statement 9 ) are of fundamental importance.

2. Contraceptive measures

2.1 Women with SLE should be counselled about the use of effective contraceptive measures (oral contraceptives, subcutaneous implants, IUD), based on their disease activity and thrombotic risk (particularly aPL status). IUD can be offered to all the patients with SLE and/or APS free of any gynaecological contraindication (1/A).

2.2 In patients with stable/inactive SLE and negative aPL, combined hormonal contraceptives can be considered (1/A). In women with positive aPL with or without definite APS, hormonal contraception (with progesterone only) must be carefully weighed against the risk of thrombosis (2/B).

3. Risk factors for reduced fertility

Women with SLE who wish to plan a pregnancy should be counselled about fertility issues, especially the adverse outcomes associated with increasing age and the use of alkylating agents (1/A). Treatment with alkylating agents should be balanced against the risk of ovarian dysfunction.

4. Preservation of fertility

Fertility preservation methods, especially GnRH analogues, should be considered for all menstruating women with SLE who are going to receive alkylating agents (2/B).

5. Assisted reproduction techniques

5.1 Assisted reproduction techniques, such as ovulation induction treatments and in vitro fertilisation protocols, can be safely used in patients with SLE with stable/inactive disease (3/C).

5.2 Patients with positive aPL/APS should receive anticoagulation (at the dosage as would be recommended during pregnancy) and/or low-dose aspirin (3/D).

6. Predictive biomarkers for maternal disease activity in SLE pregnancy

In pregnant women with SLE, assessment of disease activity (1/A) -including renal function parameters (2/B) and serological markers

(serum C3/C4, anti-dsDNA titres) (2/B) - is recommended to monitor for obstetrical adverse outcomes and disease flares.

7. Pregnancy monitoring
7.1 Women with SLE and/or APS should undergo supplementary fetal surveillance with Doppler ultrasonography and biometric parameters,

particularly in the third trimester to screen for placental insufficiency and small for gestational age fetuses (3/D).

7.2 Fetal echocardiography is recommended in cases of suspected fetal dysrhythmia or myocarditis, especially in patients with positive anti-Ro/ SSA and/or anti-La/SSB antibodies (2/C)t.

8. Drugs for the prevention and management of SLE flares during pregnancy

8.1 HCQ (1/B), oral glucocorticoids, azathioprine, ciclosporin A and tacrolimus (all 3/C) can be used to prevent or manage SLE flares during pregnancy.

8.2 Moderate-to-severe flares can be managed with additional strategies, including glucocorticoids intravenous pulse therapy, intravenous immunoglobulin and plasmapheresis (all $3 / \mathrm{C}$ ).

8.3 Mycophenolic acid, cyclophosphamide, leflunomide and methotrexate should be avoided.

9. Adjunct treatment during pregnancy

9.1 HCQ is recommended preconceptionally and throughout pregnancy for patients with SLE (2/B).

$\overline{\text { Mean (SD) Median (IQR) }}$

$10(0.2)$

$10(0)$

9.2 Women with SLE at risk of pre-eclampsia (especially those with lupus nephritis or positive aPL) should receive LDA (2/C). In women with SLE-associated APS or primary APS, combination treatment with LDA and heparin is recommended to decrease the risk of adverse pregnancy outcomes (1/A).

9.3 Supplementation with calcium, vitamin D and folic acid should be offered as in the general population (-/D). Measuring blood vitamin D levels should be considered after pregnancy is confirmed (-ID).

10. Menopause and HRT

HRT can be used for the management of severe vasomotor menopausal manifestations in SLE women with stable/inactive disease and negative aPL (1/A). The use of HRT in patients with positive aPL should be carefully weighed against the risk of thrombosis and cardiovascular disease (-/D).

11. Screening for malignancies Women with SLE and/or APS should undergo screening for malignancies similar to the general population (-/D). Women with SLE, especially those exposed to immunosuppressive drugs, are at higher risk of cervical premalignant lesions and should be monitored with vigilance (2/B).

12. HPV vaccination HPV immunisation can be considered in women with SLE and/or APS and stable/inactive disease (3/D).

For each statement or item, the LoE (range 1-3) and the GoR (range A-D) is given in parentheses (refer to online supplementary table S1). In the right-hand columns, the LoA among experts is reported as mean (SD) and median (IQR) values. A score of 10 represents the highest level of agreement.

${ }^{*}$ aPL and APS are defined according to the updated international consensus criteria. ${ }^{6}$ For aPL assays, please see the footnotes of table 2.

tThe substatement on fetal echo in women with SLE/APS and positive anti-Ro/La is rated with LoE=2 (ie, sufficient evidence for the association between anti-Ro/La and congenital heart block) but $G o R=C$ due to lack of strong evidence for the clinical implications of this association, namely for the efficacy of interventions.

anti-dsDNA, anti-double-stranded DNA antibodies; aPL, antiphospholipid antibodies; GnRH, gonadotropin-releasing hormone; GoR, grade of recommendation; HCQ, hydroxychloroquine;

HPV, human papillomavirus; HRT, hormone replacement therapy; IUD, intrauterine devices; LDA, low-dose aspirin; LoA, level of agreement; LoE, level of evidence. 
Table 2 Checklist of parameters to be considered for preconception counselling and risk stratification in women with systemic lupus erythematosus (SLE) and/or antiphospholipid syndrome (APS)

Disease-related risk factors
SLE activity/flares* (in the last 6-12 months
or at conception)

Lupus nephritis (history or active at conceptiont)

Serological (serum C3/C4, anti-dsDNA titres) activity

Previous adverse pregnancy outcome(s)

History of vascular thrombosis

SLE diagnosis

aPL profile‡

\section{Prognostic implications}

Increased risk for (i) maternal disease activity (RR 2.1 for subsequent flare during pregnancy and puerperium): ${ }^{14}$ (ii) hypertensive complications (OR 1.8 for PE) $i^{15}$ (iii) fetal morbidity and mortality (OR 5.7 for pregnancy loss, ${ }^{16} 3.5$ for IUGR $^{17} 6.5$ for preterm delivery) ${ }^{14} 1517-22$

Strong predictor of poor maternal (RR 9.0 for renal flare during/after pregnancy) ${ }^{23}$ and fetal outcome(s) (OR 7.3 for fetal loss and 18.9 for preterm delivery) 2425

Increased risk for maternal SLE flares during pregnancy (OR 5.3) ${ }^{14}$ and pregnancy loss 232627

APS: increased risk for pregnancy complications ${ }^{28-30}$

APS: increased risk (ORs ranging 3.6-12.7) for pregnancy morbidity ${ }^{31}$

APS: increased risk (OR 6.9) for pregnancy morbidity ${ }^{31} 32$

SLE: strong predictor of adverse maternal and fetal outcomes, 1925273334 especially for patients with persistent moderate-to-high aPL titres, LA and multiple aPL positivity (high-risk aPL profile)

APS: high-risk aPL profile correlates with increased risk of maternal vascular thrombotic events during pregnancy $(0 \mathrm{R} 12.1)^{35}$ (pre-)eclampsia (OR 2.3), ${ }^{36}{ }^{37}$ APS-related pregnancy morbidity (OR 9.2), ${ }^{31}$ IUGR (OR 4.7), ${ }^{36}$ preterm birth $^{38} 39$

\begin{tabular}{ll} 
Anti-Ro/SSA, anti-La/SSB antibodies & Linked to development of neonatal lupus, including a low risk (0.7-2\%) for CHB (especially if moderate-to-high anti-Ro \\
titres); & $40-43$ weak association with other pregnancy complications \\
\hline End-stage organ damage and associated & 4546
\end{tabular}

comorbidities

General risk factors

Maternal age

Arterial hypertension

Diabetes mellitus

Increased risk for pregnancy loss (OR 2.4, ${ }^{33}$ RR 2.9), ${ }^{48}$ preterm birth $^{18} 2427$ and IUGR (OR 6.8) 49

Overweight/obesity

Thyroid disease

Nicotine and alcohol use

Immunisations§

* Diagnosed by validated SLE activity indices and/or physician judgement.

tEvaluated by renal function tests (serum creatinine, blood urea nitrogen) and urinalysis (proteinuria urine sediment).

¥Includes LA, aCL IgG/lgM, a $32 \mathrm{GPI}$ IgG/lgM. The level of positivity of aCL and aß2GPI antibodies (low vs medium-high) should be defined according to the single assay's characteristics. §If negative serology, evaluate whether immunisations can be performed prior to pregnancy (eg, rubella).

aCL, anticardiolipin antibodies; aß2GPI, anti- $\beta 2$-GPI antibodies; anti-dsDNA, anti-double-stranded DNA antibodies; aPL, antiphospholipid antibodies; CHB, congenital heart block; IUGR, intrauterine growth restriction; LA, lupus anticoagulant; PE, pre-eclampsia; RR, relative risk.

discussions, the committee arrived at 12 final statements (table 1). Each member rated her/his agreement with each statement.

\section{RESULTS AND DISCUSSION \\ Scope and overarching principles}

These recommendations have been devised with the intention of helping physicians involved in the care of patients with SLE and/or APS and facilitating physician-patient communication. They recognise an implicit need for change in the mindset of health professionals, shifting from caution against pregnancy towards embracement of pregnancy. Accordingly, family planning should be discussed from the first physician-patient encounter and reinforced thereafter. Health professionals should support the patient and her family in their decisions regarding family planning by discussing individual pregnancy risks. Reports on the long-term follow-up of SLE and/or APS offspring are few, ${ }^{7-10}$ showing a reassuring picture on the health conditions of the children, with the exception of some cases of neurodevelopmental alterations ${ }^{11-13}$ that need further confirmation before they are linked to maternal disease.

\section{Recommendations}

Preconception counselling and risk stratification

Assessment of risk factors for adverse maternal and fetal outcomes in pregnant women with SLE and/or APS is crucial for preconception counselling and implementing appropriate preventive strategies and patient-tailored monitoring plan before and during pregnancy (table 2).

In SLE women (with or without APS), prematurity, preeclampsia and eclampsia/Hemolysis, Elevated Liver enzyme levels, Low Platelet count (HELLP) rates approximate $25-35 \%$, $10-15 \%$ and $1.0-1.5 \%$, respectively. ${ }^{19} \quad 24 \quad 25 \quad 44 \quad 51 \quad 52$ In APS women (primary or SLE-related), the respective frequencies approximate 25-35\%, 10-20\% and 3.0-5.0\%. ${ }^{28} 295354$

During pregnancy, risk factors associated with adverse outcomes include active/flaring SLE (OR 12.7 for pre-eclampsia/ eclampsia; ${ }^{55} 19.0$ for emergency caesarean section; ${ }^{56} 3.0$ for early fetal loss; ${ }^{20} 5.5$ for preterm delivery), ${ }^{21} 19$ active nephritis (OR 5.3 for any adverse maternal outcome), ${ }^{57}$ hypertension (OR 4.8-7.3 for pre-eclampsia; ${ }^{52}$ relative risk (RR) 1.8 for preterm birth) ${ }^{22}$ and use of glucocorticoids, especially at maintenance dose $\geq 10-20 \mathrm{mg} /$ day of prednisone equivalent (OR 3.5 for preterm birth). ${ }^{58}$ Discontinuation of hydroxychloroquine (HCQ) is related to an increased risk for SLE exacerbations during pregnancy, 243356 and a single placebo-controlled study has suggested a beneficial effect of HCQ on maternal disease activity during pregnancy. ${ }^{60}$

\section{Contraceptive measures}

Women with SLE and/or APS should be counselled about contraception, especially for the prevention of unwanted pregnancies during high disease activity periods and intake of teratogenic drugs. Effective contraceptive measures should be 
discussed with the patient by weighing the individual risk factors, including general (hypertension, obesity, tobacco use, family history of hormonal-dependent cancers) ${ }^{61}$ and diseaserelated risk factors, particularly disease activity and thrombotic risk (emphasis on antiphospholipid antibodies (aPLs)).

The intrauterine device (IUD) can be offered to all patients unless there is a gynaecological contraindication. Copper IUD can be used in any patient, while levonorgestrel-containing IUD should be considered only if the benefits of the released hormone (such as the reduction of excessive menstrual bleeding due to anticoagulation) ${ }^{62}$ outweigh the risk of thrombosis. ${ }^{61}$

The safety of the combined (oestrogen plus progestin) and progestin-only pill in SLE patients with inactive or stable active SLE and negative aPL has been demonstrated in randomised controlled trials (RCTs). ${ }^{63}$ In women with positive aPL (with or without definite APS), contraception with combined hormones (oral pill, vaginal ring, transdermal patch) should be discouraged. In young women with myocardial infarction or ischaemic stroke and positive lupus anticoagulant, the use of the combined pill increased the risk of arterial events compared with non-users. ${ }^{65}$ In fully anticoagulated patients carrying a low-risk aPL profile, oestrogens might be considered for persistent gynaecological disorders not otherwise managed. Compounds containing progestin only (pill, subcutaneous depot injections) are suitable for these women, although their use should be weighed against the risk of thrombosis. Progestin-only emergency contraception is not contraindicated in patients with SLE and/or APS.

\section{Risk factors for reduced fertility}

Few studies have assessed fertility in women with SLE and/or APS by means of hormonal levels (including the anti-Müllerian hormone) or antral follicle count (examined by ultrasound). There is no concrete evidence that the disease per se decreases fertility. ${ }^{66-69}$

However, active disease, especially lupus nephritis, and the use of immunosuppressive drugs may negatively impact on fertility. Alkylating agents such as cyclophosphamide (CYC) may cause menstrual irregularities and premature ovarian failure (POF), which is age- and dosage dependent. ${ }^{70} 71$

Similar to the general population, women with SLE and/or APS should be counselled on fertility issues, especially on the negative impact of increasing age (general tendency to postpone childbearing) and certain lifestyle exposures (tobacco use, alcohol consumption). In non-life-threatening disease, treatment with alkylating agents should be balanced against the risk of ovarian dysfunction; rather, less gonadotoxic regimens should be considered. ${ }^{72}$ In the presence of multiple risk factors for impaired fertility, ovarian reserve may be assessed in patients with SLE at a younger age than recommended for the general population. $^{73}$

\section{Fertility preservation}

Limited data are available on fertility preservation methods in menstruating women with SLE who require treatment with alkylating agents. Cryopreservation of ovarian tissue or oocytes/ embryos are poorly investigated options ${ }^{74} 75$ and require specialised centres, which may not be easily accessible.

The most extensively studied method for POF prevention in patients with SLE involves gonadotropin-releasing hormone analogues (GnRH-a), with a good safety and efficacy profile (RR 0.12). ${ }^{76} \mathrm{GnRH}-\mathrm{a}$ have been efficacious in patients with cancer. ${ }^{77}{ }^{78} \mathrm{GnRH}$-a are likely to protect against POF, but there are no data on subsequent pregnancies in patients with SLE.
They can cause menopause-like symptoms, which are fully reversible upon discontinuation. A study in childhood-onset patients with SLE aged <21 years suggested that GnRH-a should be administered 22 days before CYC is started or continued. $^{79}$ It is nevertheless recommended to start the GnRH-a prior to or concomitantly to initiation of the alkylating agent.

\section{Assisted reproduction techniques}

Evidence on the efficacy and safety of ARTs (ovulation induction therapy and in vitro fertilisation) in women with SLE and/or APS comes from observational studies. ${ }^{80-83}$ Efficacy in terms of pregnancy rate is comparable with that in the general population (up to $30 \%$ ). ARTs are generally safe if the patient has quiescent disease and is on appropriate antithrombotic treatment if aPL positive. Although it is challenging to define a single protocol, some general measures for prophylaxis in aPL-positive women undergoing ovarian stimulation can be suggested. The type (low-dose aspirin (LDA); low molecular weight heparin $(\mathrm{LMWH})$ ) and dosage (prophylactic vs full anticoagulant) of antithrombotic treatment should be recommended as during pregnancy according to the individual risk profile. LDA should be stopped three days before egg retrieval and resumed the following day. Patients taking LMWH should stop it at least 12 hours prior to the procedure and resume it the very same day as long as there is no bleeding. Patients with positive aPL who are not taking LDA during the ovarian stimulation period should start LDA on the day of the embryo transfer, usually in combination with LMWH (which will be continued during pregnancy).

Ovarian hyperstimulation syndrome can be avoided by milder hormonal stimulation or GnRH antagonist protocol. ${ }^{84}$ The use of the 'natural cycle' method is another option, although associated with a lower rate of induced pregnancy. The ART induction protocol should be tailored to the individual patient, balancing the safety and effectiveness of the procedure.

\section{Predictive biomarkers for maternal disease activity in SLE pregnancy}

Active SLE during pregnancy, assessed by validated disease activity indices ${ }^{22} 56$ and/or physician global assessment, ${ }^{20}$ is associated with increased risk for maternal and/or fetal complications (see also paragraph on Preconception counselling and risk stratification). Pregnancy-specific SLE activity indices have been developed and validated for their sensitivity in detecting changes in disease activity and diagnosing flares (see online supplementary table S4). ${ }^{85} 86$ Physicians should be aware of pregnancy physiological changes that can resemble SLE symptoms and signs. ${ }^{87}$ Renal activity correlates with adverse pregnancy outcomes and should be monitored by means of urine protein excretion, urine sediment analysis (glomerular haematuria, urinary casts) and serum creatinine level/glomerular filtration rate. $^{33} 4952$ Serological markers are useful in monitoring SLE activity and in the differentiation between disease exacerbation (declining serum $\mathrm{C} 3 / \mathrm{C} 4$ levels (even within the normal range) and/or increasing anti-double stranded DNA titres) and preeclampsia. ${ }^{88} 89$ Smaller increases in serum C3 levels from pregnancy onset to the second or third trimester ${ }^{19}$ as well as serological activity (as defined above) that develops during pregnancy, especially in the context of clinical SLE activity, have been associated with increased risk for pregnancy loss, ${ }^{19} 90$ intrauterine growth restriction (IUGR) ${ }^{91}$ and preterm birth. 1948899092 
Box 1 Ultrasonographic fetal surveillance recommended

for pregnant women with systemic lupus erythematosus and/or antiphospholipid syndrome

- Routine ultrasonographic screening

- First trimester (11-14 weeks of gestation).

- Second trimester (with Doppler, preferably at 20-24 weeks of gestation).

- Supplementary fetal surveillance in the third trimester at monthly intervals

- Doppler sonography of the umbilical artery, uterine arteries, ductus venosus and middle cerebral artery (particularly in fetuses that have been identified to suffer from early intrauterine growth restriction (IUGR), ie, prior to 34 weeks of gestation).

- In cases of late IUGR (diagnosed after 34 weeks), reduced abdominal circumference growth velocity and/or a reduced cerebroplacental ratio at Doppler investigation was shown to identify fetuses at higher risk of poor perinatal outcome (Doppler of the umbilical artery alone is insufficient).

\section{Pregnancy monitoring}

Pregnant women with SLE and/or APS should follow the local protocols applied to pregnancies at high risk for hypertensive disorders and/or placental insufficiency, adjusting the frequency and modality of fetal surveillance according to the maternal and/or fetal status (box 1). Fetal surveillance based on biometric and Doppler findings during the third trimester, and particularly the distinction between early and late IUGR, helps to better tailor the time of delivery and reduce perinatal morbidity and mortality. ${ }^{93-97}$ Umbilical and uterine arteries Doppler sonography at 20-24 weeks has good negative predictive value but modest positive predictive value (especially in the absence of biometric signs of fetal growth restriction later in pregnancy) for placental-associated pregnancy disorders such as preeclampsia and IUGR. The mode (vaginal vs caesarean section) and timing of delivery are influenced by maternal (hypertensive disorders, anticoagulation status) as well as fetal conditions during pregnancy.

Fetal echocardiography is indicated if there is suspected fetal dysrhythmia or myocarditis, especially in the context of positive maternal anti-Ro/SSA or anti-La/SSB antibodies. Other tests (electrocardiogram plus Holter monitor, magnetocardiography, gated-pulsed Doppler technique, velocity-based fetal kinetocardiogram) might detect subtle signs of the development of congenital heart block (CHB), but are not currently recommended as standard practice. ${ }^{98} \mathrm{CHB}$ associated with anti-Ro/SSA and/or anti-La/SSB has $16 \%$ recurrence rate in women with a previously affected child; therefore, it is recommended to perform serial fetal echocardiograms weekly from 16 weeks of gestation onwards. ${ }^{98}$ Considering the low risk $(0.7-2 \%)$ for $\mathrm{CHB}$ in women with no previous $\mathrm{CHB}$, it is unclear whether intensive monitoring (weekly/biweekly between 16 and 26 weeks of gestation and less frequently afterwards $)^{98}$ in the general population of anti-Ro/La-positive women is cost-effective. Moreover, there is no proven efficacy of protocols for the prevention or treatment of complete CHB. ${ }^{99} 100$ The efficacy of maternal fluorinated steroids has not been established in large cohorts ${ }^{101-104}$ despite initial reports of favourable effects in cases of incomplete $\mathrm{CHB}$, cardiomyopathy, endocardial fibroelastosis and hydrops fetalis. ${ }^{99}$ Given the potential of fluorinated steroids for major maternal and fetal side effects, the benefit for fetuses with $\mathrm{CHB}$ should be stratified according to the presence of risk factors for adverse outcome. ${ }^{99}$ Despite its unproven benefit, the current practice of intensive surveillance for $\mathrm{CHB}$ onset in women with positive anti-Ro/SSA and/or anti-La/SSB antibodies and no previous child affected by CHB carries no risk and is well accepted by the mothers. ${ }^{105}$

\section{Drugs for prevention and management of SLE flares during pregnancy}

A single randomised, placebo-controlled study ${ }^{60}$ as well as nonrandomised evidence ${ }^{24} 3356$ supports the beneficial role of HCQ in controlling disease activity and preventing flare-ups during pregnancy. Uncontrolled studies suggest an acceptable benefit/risk ratio of oral glucocorticoids, ${ }^{22} 106$ azathioprine ${ }^{59} 107$ and calcineurin inhibitors (ciclosporin A, tacrolimus) ${ }^{108} 109$ in controlling SLE activity during pregnancy. In moderate-to-severe flares, additional modalities can be considered, such as high-dose glucocorticoids (including pulse intravenous therapy), ${ }^{110} 111$ intravenous immunoglobulin ${ }^{20} 22$ and plasmapheresis (may be also used in refractory nephrotic syndrome). ${ }^{112}{ }^{113}$ CYC should not be administered during the first trimester of pregnancy due to risk for fetal loss (OR 25.5) ${ }^{20114}$ and should be reserved only for the management of severe, life-threatening or refractory SLE manifestations during the second or third trimester. Available data are not sufficient to evaluate the risk of using belimumab in pregnancy ${ }^{115}$ and the drug should not be used unless the benefit outweighs the risk to the fetus. Mycophenolic acid, methotrexate and leflunomide should be avoided due to known or possible teratogenicity. ${ }^{116}$ To this end, collaborative groups have developed recommendations for the use of antirheumatic drugs before and during pregnancy and lactation. ${ }^{111} 117118$

\section{Adjunct treatment during pregnancy}

Use of HCQ is recommended in women with SLE preconceptionally and throughout pregnancy. ${ }^{33} 5660$ A beneficial role has also been suggested for APS pregnancies, ${ }^{119-121}$ but at present there is insufficient data to recommend its routine use in these patients. HCQ may reduce the odds of $\mathrm{CHB}$ occurrence in fetuses exposed to maternal anti-Ro/SSA antibodies, especially in mothers who already had a child with $\mathrm{CHB} .{ }^{40} 122$

The protective role of LDA against preterm and severe preeclampsia has been established in non-autoimmune patients. ${ }^{123} 124$ Accordingly, women with SLE at higher risk of pre-eclampsia including those with lupus nephritis or positive aPL will benefit from LDA, preferably given preconceptionally or no later than gestational week $16 .^{123} 124$

In women with definite obstetric APS, combination treatment with LDA and heparin is recommended to decrease the risk of adverse pregnancy outcomes. ${ }^{16}{ }^{125-127}$ Statistically significant results have been demonstrated only for unfractionated heparin in RCTs. However, LMWH is preferable for practical reasons and has shown comparable efficacy in prospective studies. ${ }^{128} 129$ Moreover, patients with positive aPL but with no definite classification of APS will benefit from combination therapy if they are considered at moderate to high risk of maternal and fetal complications (see online supplementary table S5).

In addition, other regimens such as prednisolone $10 \mathrm{mg} /$ day in the first trimester, intravenous immunoglobulin or plasmapheresis can be considered for selected patients with APS (refractory obstetric APS, women with previous thrombosis, particularly previous or new cerebrovascular events, women with triple aPL positivity). ${ }^{119} 130-133$ 


\section{Box 2 Points to consider and research agenda}

- Reproductive issues are of paramount importance for women with systemic lupus erythematosus (SLE) and/or antiphospholipid syndrome (APS) and should be addressed on a regular basis by healthcare providers.

- Preconception counselling and risk stratification are essential for prevention of unwanted complications during pregnancy.

- The use of hormonal contraception or replacement therapy is feasible but must be weighed against the individual risk of thrombosis.

- The preservation of fertility should be mentioned while counselling about lifestyles and considered in the treatment choice. Validated protocols for assisted reproduction techniques in patients with SLE and/or APS are needed.

- Predictive biomarkers for maternal disease activity during SLE pregnancy should be expanded with particular focus on the prediction of pre-eclampsia.

- Pregnancy monitoring in SLE and/or APS women should aim at the identification of placental insufficiency with fetal growth restriction in order to decide the best timing for delivery and reduce the risk of perinatal morbidity and mortality.

- The cost-effectiveness of intensive surveillance with fetal echocardiography in patients with positive anti-Ro/SSA and anti-La/SSB antibodies and no previous child with congenital heart block remains to be established.

- Hydroxychloroquine is beneficial during pregnancy to reduce the risk of SLE flares and of poor obstetrical outcomes. More data are needed to support its benefit in APS pregnancies.

- The benefits of cancer surveillance and prevention of gynaecological malignancies need to be communicated to patients.

As recommended in the general population, supplementation with calcium, vitamin D and folic acid should be offered to patients with SLE and/or APS, with particular consideration to those with low circulating levels of $25-\mathrm{OH}$ vitamin $\mathrm{D}$ in the first trimester of gestation and receiving glucocorticoids and/or heparin for their detrimental effects on bone mass.

\section{Menopause and hormone replacement therapy}

The efficacy and safety of hormone replacement therapy (HRT) (oestrogen plus progestin) in selected patients with SLE has been illustrated in RCTs. ${ }^{134-136}$ Benefit was demonstrated mainly in vasomotor and other hypoestrogenism symptoms. No significantly increased risk of severe lupus exacerbations during 12-24 months of HRT was found, although there was a modest increase in mild-to-moderate flares. ${ }^{132}$ There was no increased risk of thrombosis and cardiovascular events, although one of the RCTs included only patients with negative aPL and no previous cardiovascular events ${ }^{132}$ and another did not detail the aPL profile. ${ }^{63}$ Two cohort studies with long-term follow-up did not report significantly increased risk of cardiovascular events during HRT, ${ }^{137} 138$ although limitations in power and design preclude firm conclusions. Consequently, HRT should be reserved for the management of severe and disabling vasomotor menopausal symptoms, preferably in SLE women with stable/ inactive disease and negative aPL. In patients with positive aPL, the use of HRT should be carefully weighed against thrombotic and cardiovascular risks. If menopause symptoms necessitate
HRT, it seems reasonable to start it as early as possible to gain an added benefit for bone protection. ${ }^{139}$ Optimal duration of HRT in patients with SLE and/or APS is not known, but it seems reasonable to recommend it for the shortest possible duration. ${ }^{140} 141$

\section{Screening for malignancies}

Women with SLE are not at increased risk of breast, ovarian and endometrial cancer compared with the general population, ${ }^{142} 143$ and, therefore, should follow the current population screening protocols for these malignancies. Conversely, women with SLE are at higher risk of cervical dysplasia (but not cervical cancer),${ }^{144-147}$ vagina and vulva cancers, ${ }^{142}{ }^{147}$ likely associated with human papillomavirus (HPV) infection. Women with SLE exposed to immunosuppressive drugs, particularly CYC in a cumulative dose-dependent fashion, are at higher risk of cervical dysplasia. ${ }^{148-151}$ The suggested timing for Papanicolaou (PAP) smear examination would be once a year in heavily immunosuppressed patients or according to the local screening programme in low-risk patients. Subgroups of women with SLE (Caucasian, younger age, lower education, high SLE damage) may be at risk for poorer adherence to screening programmes. ${ }^{152} 153$

\section{HPV vaccination}

HPV vaccination is currently offered to female and male adolescents for preventing precancerous growths and cancer in the cervix and in the genital area. There are reports of venous thromboembolic events (VTEs) associated with the quadrivalent HPV vaccine. However, of the 31 cases $(0.2 / 100000$ doses vaccine) with documented VTE, 90\% had a known risk factor for VTE (APS in two cases). ${ }^{154}$

Prospective studies have demonstrated efficacy and safety of HPV vaccination in patients with SLE, ${ }^{155} 156$ although seroconversion rates may be lower in patients receiving steroids and immunosuppressive agents. A few cases of severe SLE flares or abrupt SLE onset after HPV vaccination have been reported. ${ }^{157-159}$ In accordance with the EULAR recommendations, ${ }^{160}$ we recommend that HPV vaccination be offered to young women with stable/inactive SLE and/or APS, according to local protocols, with particular caution in those with high-risk aPL profile.

The points to consider and the research agenda suggested by the Task Force Members are reported in box 2.

\section{Author affiliations}

${ }^{1}$ Department of Clinical and Experimental Sciences, University of Brescia, Brescia, Italy

${ }^{2}$ Unit of Rheumatology and Clinical Immunology, Spedali Civili, Brescia, Italy ${ }^{3}$ Department of Rheumatology, Clinical Immunology and Allergy, University of Crete Medical School, Heraklion, Greece

${ }^{4}$ The Zabludowicz Center for Autoimmune Diseases, Sheba Medical Center,

Tel Hashomer, Israel

${ }^{5}$ The Faculty of Medicine, Tel Aviv University, Israel

${ }^{6}$ Royal National Hospital For Rheumatic Diseases, Bath, UK

${ }^{7}$ Department of Autoimmune Diseases, Hospital Clínic, Barcelona, Catalonia, Spain

${ }^{8} \mathrm{AP}$-HP, Hôpital Cochin, Centre de référence maladies auto-immunes et systémiques rares, Paris, France

${ }^{9}$ Université Paris Descartes-Sorbonne Paris Cité, Paris, France

${ }^{10}$ Rheumatology Unit, Department of Medicine, University of Padua, Italy

${ }^{11}$ Policlinic of Rheumatology, Hiller Research Unit, University Clinic Duesseldorf,

Heinrich-Heine-University Duesseldorf, Duesseldorf, Germany

${ }^{12}$ Department of Rheumatology, Immunology and Allergology, University Hospital of

Bern, Bern, Switzerland

${ }^{13}$ Unidade de Doenças Auto-imunes—Serviço Medicina Interna 7.2, Hospital Curry

Cabral/Centro Hospitalar Lisboa Central, NEDAI/SPMI, Lisboa, Portugal

${ }^{14}$ Lupus Research Unit, The Rayne Institute, St. Thomas Hospital, London, UK

${ }^{15}$ Department of Rheumatology, Dubai Hospital, Dubai, United Arab Emirates

${ }^{16}$ EULAR PARE Patient Research Partner, London, UK 
${ }^{17}$ Unit of Obstetrics and Gynaecology, Spedali Civili, Brescia, Italy

${ }^{18}$ EULAR PARE Patient Research Partner, Rome, Italy

${ }^{19}$ Department of Clinical Sciences and Community Health, University of Milan, Istituto Auxologico Italiano, Milan, Italy

${ }^{20}$ Rheumatology Unit, Department of Clinical and Experimental Medicine, University of Pisa, Pisa, Italy

${ }^{21}$ Neonatology and Neonatal Intensive Care Unit, Spedali Civili, Brescia, Italy

${ }^{22}$ Norwegian National Advisory Unit on Pregnancy and Rheumatic Diseases,

St. Olavs Hospital, Trondheim University Hospital, Trondheim, Norway

${ }^{23}$ Department of Rheumatology, Iuliu Hatieganu University of Medicine and

Pharmacy, Cluj-Napoca, Romania

${ }^{24}$ Department of Obstetrics and Gynaecology, University Hospital of Bern, Inselspital, Switzerland

${ }^{25}$ Rheumatology Unit, Department of Medicine, Solna, Karolinska Institutet, Karolinska University Hospital, Stockholm, Sweden

${ }^{26}$ Rheumatology Unit, Joint Academic Rheumatology Programme, 1st Department of Propaedeutic Internal Medicine Athens, National and Kapodistrian University of Athens, Athens, Greece

${ }^{27}$ Department of Rheumatology, Istanbul Bilim University, Istanbul Florence Nightingale Hospital, Esentepe-Istanbul, Turkey

${ }^{28} 4$ th Department of Internal Medicine, 'Attikon' University Hospital, Medical School, University of Athens, Athens, Greece

${ }^{29}$ Joint Academic Rheumatology Program, National and Kapodestrian University of Athens, Athens, Greece

Acknowledgements The committee wishes to acknowledge the support of the EULAR Standing Committee on Clinical Affairs. The committee also expresses its sincere appreciation and gratitude to the EULAR Secretariat and especially to Patrizia Jud, executive assistant, for outstanding organisation.

Contributors LA and GKB contributed equally to the article. DB and AT share senior authorship. All the authors contributed to discussion of the results of the systematic literature review (performed by LA and GKB) and to the review of the article.

Competing interests None declared.

Provenance and peer review Not commissioned; externally peer reviewed.

Open Access This is an Open Access article distributed in accordance with the Creative Commons Attribution Non Commercial (CC BY-NC 4.0) license, which permits others to distribute, remix, adapt, build upon this work non-commercially, and license their derivative works on different terms, provided the original work is properly cited and the use is non-commercial. See: http://creativecommons.org/ licenses/by-nc/4.0/

\section{REFERENCES}

1 Østensen M. New insights into sexual functioning and fertility in rheumatic diseases. Best Pract Res Clin Rheumatol 2004;18:219-32.

2 Ntali S, Damjanov N, Drakakis P, et al. Women's health and fertility, family planning and pregnancy in immune-mediated rheumatic diseases: a report from a south-eastern European Expert Meeting. Clin Exp Rheumatol 2014:32: 959-68.

3 Kavanaugh A, Cush JJ, Ahmed MS, et al. Proceedings from the American College of Rheumatology Reproductive Health Summit: the management of fertility, pregnancy, and lactation in women with autoimmune and systemic inflammatory diseases. Arthritis Care Res (Hoboken) 2015;67:313-25.

4 Østensen M, Andreoli L, Brucato A, et al. State of the art: reproduction and pregnancy in rheumatic diseases. Autoimmun Rev 2015;14:376-86.

5 van der Heijde D, Aletaha D, Carmona L, et al. 2014 Update of the EULAR standardised operating procedures for EULAR-endorsed recommendations. Ann Rheum Dis 2015;74:8-13.

6 Miyakis S, Lockshin MD, Atsumi T, et al. International consensus statement on an update of the classification criteria for definite antiphospholipid syndrome (APS). J Thromb Haemost 2006:4:295-306.

7 Nalli C, lodice A, Andreoli L, et al. The effects of lupus and antiphospholipid antibody syndrome on foetal outcomes. Lupus 2014;23:507-17.

8 Andreoli L, Fredi M, Nalli C, et al. Pregnancy implications for systemic lupus erythematosus and the antiphospholipid syndrome. J Autoimmun 2012;38: J197-208.

9 Parke A. Drug exposure, pregnancy outcome and fetal and childhood development occurring in the offspring of mothers with systemic lupus erythematosus and other chronic autoimmune diseases. Lupus 2006;15:808-13.

10 Vinet $E_{\text {, }}$ Pineau CA, Clarke AE, et al. Neurodevelopmental disorders in children born to mothers with systemic lupus erythematosus. Lupus 2014;23:1099-104.

11 Nalli C, lodice A, Andreoli L, et al. Children born to SLE and APS mothers. Lupus 2014:23:1246-8

12 Mekinian A, Lachassinne E, Nicaise-Roland $P$, et al. European registry of babies born to mothers with antiphospholipid syndrome. Ann Rheum Dis 2013;72:217-22.
13 Vinet É, Pineau CA, Clarke AE, et al. Increased risk of autism spectrum disorders in children born to women with systemic lupus erythematosus: results from a large population-based cohort. Arthritis Rheumatol 2015;67:3201-8.

14 Yang $\mathrm{H}$, Liu H, Xu D, et al. Pregnancy-related systemic lupus erythematosus: clinical features, outcome and risk factors of disease flares - a case control study. PLOS ONE 2014;9:e104375.

15 Kwok LW, Tam LS, Zhu T, et al. Predictors of maternal and fetal outcomes in pregnancies of patients with systemic lupus erythematosus. Lupus 2011;20:829-36.

16 Roberge $S$, Giguère $Y$, Villa $P$, et al. Early administration of low-dose aspirin for the prevention of severe and mild preeclampsia: a systematic review and meta-analysis. Am J Perinatol 2012;29:551-6.

17 Chen TK, Gelber AC, Witter FR, et al. Renal biopsy in the management of lupus nephritis during pregnancy. Lupus 2015;24:147-54.

18 Le Thi Huong D, Wechsler B, Piette JC, et al. Pregnancy and its outcome in systemic lupus erythematosus. QJM 1994;87:721-9.

19 Buyon JP, Kim MY, Guerra MM, et al. Predictors of pregnancy outcomes in patients with lupus: a cohort study. Ann Intern Med 2015;163:153-63.

20 Clowse ME, Magder LS, Witter F, et al. The impact of increased lupus activity on obstetric outcomes. Arthritis Rheum 2005;52:514-21.

21 Mok MY, Leung PY, Lao TH, et al. Clinical predictors of fetal and maternal outcome in Chinese patients with systemic lupus erythematosus. Ann Rheum Dis 2004;63:1705-6.

22 Chakravarty EF, Colón I, Langen ES, et al. Factors that predict prematurity and preeclampsia in pregnancies that are complicated by systemic lupus erythematosus. Am J Obstet Gynecol 2005;192:1897-904.

23 Imbasciati E, Tincani A, Gregorini G, et al. Pregnancy in women with pre-existing lupus nephritis: predictors of fetal and maternal outcome. Nephrol Dial Transplant 2009;24:519-25.

24 Al Arfaj AS, Khalil N. Pregnancy outcome in 396 pregnancies in patients with SLE in Saudi Arabia. Lupus 2010;19:1665-73.

25 Smyth A, Oliveira GH, Lahr BD, et al. A systematic review and meta-analysis of pregnancy outcomes in patients with systemic lupus erythematosus and lupus nephritis. Clin J Am Soc Nephrol 2010;5:2060-8.

26 Shibata S, Sasaki T, Hirabayashi Y, et al. Risk factors in the pregnancy of patients with systemic lupus erythematosus: association of hypocomplementaemia with poor prognosis. Ann Rheum Dis 1992;51:619-23.

27 Cortes-Hernandez J, Ordi-Ros J, Paredes F, et al. Clinical predictors of fetal and maternal outcome in systemic lupus erythematosus: a prospective study of 103 pregnancies. Rheumatology (Oxford) 2002;41:643-50.

28 Bouvier S, Cochery-Nouvellon E, Lavigne-Lissalde G, et al. Comparative incidence of pregnancy outcomes in treated obstetric antiphospholipid syndrome: the NOH-APS observational study. Blood 2014;123:404-13.

29 Jeremic K, Stefanovic A, Dotlic J, et al. Neonatal outcome in pregnant patients with antiphospholipid syndrome. J Perinat Med 2015;43:761-8.

30 Girón-González JA, García del Río E, Rodríguez C, et al. Antiphospholipid syndrome and asymptomatic carriers of antiphospholipid antibody: prospective analysis of 404 individuals. J Rheumatol 2004;31:1560-7.

31 Ruffatti A, Tonello M, Visentin MS, et al. Risk factors for pregnancy failure in patients with anti-phospholipid syndrome treated with conventional therapies: a multicentre, case-control study. Rheumatology (Oxford) 2011:50:1684-9.

32 Danowski A, de Azevedo MN, de Souza Papi JA, et al. Determinants of risk for venous and arterial thrombosis in primary antiphospholipid syndrome and in antiphospholipid syndrome with systemic lupus erythematosus. J Rheumatol 2009;36:1195-9.

33 Clowse ME, Magder L, Witter $F$, et al. Hydroxychloroquine in lupus pregnancy. Arthritis Rheum 2006;54:3640-7.

34 Lockshin MD, Kim M, Laskin CA, et al. Prediction of adverse pregnancy outcome by the presence of lupus anticoagulant, but not anticardiolipin antibody, in patients with antiphospholipid antibodies. Arthritis Rheum 2012;64:2311-18.

35 Stone S, Hunt BJ, Khamashta MA, et al. Primary antiphospholipid syndrome in pregnancy: an analysis of outcome in a cohort of 33 women treated with a rigorous protocol. J Thromb Haemost 2005:3:243-5.

36 Abou-Nassar K, Carrier M, Ramsay T, et al. The association between antiphospholipid antibodies and placenta mediated complications: a systematic review and meta-analysis. Thromb Res 2011;128:77-85.

37 Chauleur C, Galanaud JP, Alonso S, et al. Observational study of pregnant women with a previous spontaneous abortion before the 10th gestation week with and without antiphospholipid antibodies. J Thromb Haemost 2010;8:699-706.

38 Ruffatti A, Calligaro A, Hoxha A, et al. Laboratory and clinical features of pregnant women with antiphospholipid syndrome and neonatal outcome. Arthritis Care Res (Hoboken) 2010;62:302-7.

39 Simchen MJ, Dulitzki M, Rofe $G$, et al. High positive antibody titers and adverse pregnancy outcome in women with antiphospholipid syndrome. Acta Obstet Gynecol Scand 2011;90:1428-33.

40 Izmirly PM, Costedoat-Chalumeau N, Pisoni CN, et al. Maternal use of hydroxychloroquine is associated with a reduced risk of recurrent anti-SSA/ 
Ro-antibody-associated cardiac manifestations of neonatal lupus. Circulation 2012;126:76-82.

41 Brucato A, Frassi M, Franceschini F, et al. Risk of congenital complete heart block in newborns of mothers with anti-Ro/SSA antibodies detected by counterimmunoelectrophoresis: a prospective study of 100 women. Arthritis Rheum 2001;44:1832-5.

42 Cimaz R, Spence DL, Hornberger L, et al. Incidence and spectrum of neonatal lupus erythematosus: a prospective study of infants born to mothers with anti-Ro autoantibodies. J Pediatr 2003;142:678-83.

43 Costedoat-Chalumeau N, Amoura Z, Lupoglazoff JM, et al. Outcome of pregnancies in patients with anti-SSA/Ro antibodies: a study of 165 pregnancies, with special focus on electrocardiographic variations in the children and comparison with a control group. Arthritis Rheum 2004;50:3187-94.

44 Barnado A, Wheless L, Meyer AK, et al. Pregnancy outcomes among African-American patients with systemic lupus erythematosus compared with controls. Lupus Sci Med 2014;1:e00020.

45 Andrade RM, McGwin G Jr, Alarcón GS, et al. Predictors of post-partum damage accrual in systemic lupus erythematosus: data from LUMINA, a multiethnic US cohort (XXXVIII). Rheumatology (Oxford) 2006:45:1380-4.

46 LV J, Wang W, Li Y. Clinical outcomes and predictors of fetal and maternal consequences of pregnancy in lupus nephritis patients. Int Urol Nephrol 2015:47:1379-85.

47 Moore LE. Recurrent risk of adverse pregnancy outcome. Obstet Gynecol Clin North Am 2008:35:459-71.

48 Borella $E$, Lojacono $A$, Gatto $M$, et al. Predictors of maternal and fetal complications in SLE patients: a prospective study. Immunol Res 2014;60:170-6.

49 Molad Y, Borkowski T, Monselise A, et al. Maternal and fetal outcome of lupus pregnancy: a prospective study of 29 pregnancies. Lupus 2005;14:145-51.

50 Stagnaro-Green A, Akhter E, Yim C, et al. Thyroid disease in pregnant women with systemic lupus erythematosus: increased preterm delivery. Lupus 2011;20:690-9.

51 Shand AW, Algert CS, March L, et al. Second pregnancy outcomes for women with systemic lupus erythematosus. Ann Rheum Dis 2013;72:547-51.

52 Bramham K, Hunt BJ, Bewley S, et al. Pregnancy outcomes in systemic lupus erythematosus with and without previous nephritis. J Rheumatol 2011;38:1906-13.

53 Cervera R, Piette JC, Font J, et al. Antiphospholipid syndrome: clinical and immunologic manifestations and patterns of disease expression in a cohort of 1,000 patients. Arthritis Rheum 2002;46:1019-27.

54 Alijotas-Reig J, Ferrer-Oliveras R, Ruffatti $A$, et al. The European Registry on Obstetric Antiphospholipid Syndrome (EUROAPS): a survey of 247 consecutive cases. Autoimmun Rev 2015;14:387-95.

55 Liu LL, Jiang Y, Wang LN, et al. Efficacy and safety of mycophenolate mofetil versus cyclophosphamide for induction therapy of lupus nephritis: a meta-analysis of randomized controlled trials. Drugs 2012;72:1521-33.

$56 \mathrm{Koh} J \mathrm{H}$, Ko HS, Kwok SK, et al. Hydroxychloroquine and pregnancy on lupus flares in Korean patients with systemic lupus erythematosus. Lupus 2015;24:210-17.

57 Wagner SJ, Craici I, Reed D, et al. Maternal and foetal outcomes in pregnant patients with active lupus nephritis. Lupus 2009;18:342-7.

58 Clark CA, Spitzer KA, Nadler JN, et al. Preterm deliveries in women with systemic lupus erythematosus. J Rheumatol 2003;30:2127-32.

59 Fischer-Betz R, Specker C, Brinks R, et al. Low risk of renal flares and negative outcomes in women with lupus nephritis conceiving after switching from mycophenolate mofetil to azathioprine. Rheumatology (Oxford) 2013;52: 1070-6.

60 Levy RA, Vilela VS, Cataldo MJ, et al. Hydroxychloroquine (HCQ) in lupus pregnancy: double-blind and placebo-controlled study. Lupus 2001;10:401-4.

61 WHO Guidelines Approved by the Guidelines Review Committee. Medical Eligibility Criteria for Contraceptive Use: A WHO Family Planning Cornerstone. Geneva: World Health Organization, 2010. http://apps.who.int/iris/bitstream/10665/ 181468/1/9789241549158 eng.pdf

62 Pisoni CN, Cuadrado MJ, Khamashta MA, et al. Treatment of menorrhagia associated with oral anticoagulation: efficacy and safety of the levonorgestrel releasing intrauterine device (Mirena coil). Lupus 2006;15:877-80.

63 Petri M, Kim MY, Kalunian KC, et al. Combined oral contraceptives in women with systemic lupus erythematosus. N Engl J Med 2005;353:2550-8.

64 Sánchez-Guerrero J, Uribe AG, Jiménez-Santana L, et al. A trial of contraceptive methods in women with systemic lupus erythematosus. $N$ Engl J Med 2005;353:2539-49.

65 Urbanus RT, Siegerink B, Roest M, et al. Antiphospholipid antibodies and risk of myocardial infarction and ischaemic stroke in young women in the RATIO study: a case-control study. Lancet Neurol 2009;8:998-1005.

66 Clowse ME, Chakravarty E, Costenbader $\mathrm{KH}$, et al. Effects of infertility, pregnancy loss, and patient concerns on family size of women with rheumatoid arthritis and systemic lupus erythematosus. Arthritis Care Res (Hoboken) 2012;64:668-74.

67 Ekblom-Kullberg S, Kautiainen $\mathrm{H}$, Alha $\mathrm{P}$, et al. Reproductive health in women with systemic lupus erythematosus compared to population controls. Scand J Rheumatol 2009;38:375-80.
68 Morel N, Bachelot A, Chakhtoura Z, et al. Study of anti-Müllerian hormone and its relation to the subsequent probability of pregnancy in 112 patients with systemic lupus erythematosus, exposed or not to cyclophosphamide. J Clin Endocrinol Metab 2013:98:3785-92

69 Velarde-Ochoa Mdel C, Esquivel-Valerio JA, Vega-Morales D, et al. Anti-Müllerian hormone in reproductive age women with systemic lupus erythematosus. Reumatol Clin 2015;11:78-82

70 Boumpas DT, Austin HA III, Vaughan EM, et al. Risk for sustained amenorrhea in patients with systemic lupus erythematosus receiving intermittent pulse cyclophosphamide therapy. Ann Intern Med 1993;119:366-9.

71 Ioannidis JP, Katsifis GE, Tzioufas AG, et al. Predictors of sustained amenorrhea from pulsed intravenous cyclophosphamide in premenopausal women with systemic lupus erythematosus. J Rheumatol 2002;29:2129-35.

72 Houssiau FA, Vasconcelos C, D'Cruz D, et al. The 10-year follow-up data of the Euro-Lupus Nephritis Trial comparing low-dose and high-dose intravenous cyclophosphamide. Ann Rheum Dis 2010;69:61-4

73 Practice Committee of American Society for Reproductive Medicine. Diagnostic evaluation of the infertile female: a committee opinion. Fertil Steril 2015;103: e44-50.

74 Henes $\mathrm{M}$, Henes JC, Neunhoeffer $\mathrm{E}$, et al. Fertility preservation methods in young women with systemic lupus erythematosus prior to cytotoxic therapy: experiences from the FertiPROTEKT network. Lupus 2012;21:953-8.

75 Elizur SE, Chian RC, Pineau CA, et al. Fertility preservation treatment for young women with autoimmune diseases facing treatment with gonadotoxic agents. Rheumatology (Oxford) 2008;47:1506-9.

76 Ben-Aharon I, Gafter-Gvili A, Leibovici L, et al. Pharmacological interventions for fertility preservation during chemotherapy: a systematic review and meta-analysis. Breast Cancer Res Treat 2010:122:803-11.

77 Blumenfeld Z, Zur H, Dann EJ. Gonadotropin-releasing hormone agonis cotreatment during chemotherapy may increase pregnancy rate in survivors. Oncologist 2015;20:1283-9.

78 Moore HC, Unger JM, Phillips KA, et al. Goserelin for ovarian protection during breast-cancer adjuvant chemotherapy. N Engl J Med 2015;372:923-32.

79 Brunner HI, Silva CA, Reiff A, et al. Randomized, double-blind, dose-escalation trial of triptorelin for ovary protection in childhood-onset systemic lupus erythematosus. Arthritis Rheumatol 2015;67:1377-85.

80 Guballa N, Sammaritano L, Schwartzman S, et al. Ovulation induction and in vitro fertilization in systemic lupus erythematosus and antiphospholipid syndrome. Arthritis Rheum 2000:43:550-6.

81 Huong DL, Wechsler B, Vauthier-Brouzes D, et al. Importance of planning ovulation induction therapy in systemic lupus erythematosus and antiphospholipid syndrome: a single center retrospective study of 21 cases and 114 cycles. Semin Arthritis Rheum 2002;32:174-88.

82 Ragab $A$, Barakat $R$, Ragheb $M$, et al. Subfertility treatment in women with systemic lupus erythematosus. J Obstet Gynaecol 2012;32:569-71.

83 Orquevaux P, Masseau A, Le Guern V, et al. [In vitro fertilization and systemic lupus erythematosus or antiphospholipid syndrome: an update]. Rev Med Interne 2015:36:154-8

84 Bellver J, Pellicer A. Ovarian stimulation for ovulation induction and in vitro fertilization in patients with systemic lupus erythematosus and antiphospholipid syndrome. Fertil Steril 2009;92:1803-10.

85 Ruiz-Irastorza G, Khamashta MA, Gordon C, et al. Measuring systemic lupus erythematosus activity during pregnancy: validation of the lupus activity index in pregnancy scale. Arthritis Rheum 2004;51:78-82.

86 Yee CS, Akil M, Khamashta M, et al. The BILAG2004-Pregnancy index is reliable for assessment of disease activity in pregnant SLE patients. Rheumatology (Oxford) 2012;51:1877-80.

87 Gordon C. Pregnancy and autoimmune diseases. Best Pract Res Clin Rheumatol 2004:18:359-79.

88 Buyon JP, Cronstein BN, Morris M, et al. Serum complement values (C3 and C4) to differentiate between systemic lupus activity and pre-eclampsia. Am J Med 1986;81:194-200

89 Tomer $\mathrm{Y}$, Viegas OA, Swissa $\mathrm{M}$, et al. Levels of lupus autoantibodies in pregnant SLE patients: correlations with disease activity and pregnancy outcome. Clin Exp Rheumatol 1996;14:275-80.

90 Clowse ME, Magder LS, Petri M. The clinical utility of measuring complement and anti-dsDNA antibodies during pregnancy in patients with systemic lupus erythematosus. J Rheumatol 2011;38:1012-16.

91 Kobayashi N, Yamada H, Kishida T, et al. Hypocomplementemia correlates with intrauterine growth retardation in systemic lupus erythematosus. Am J Reprod Immunol 1999;42:153-9.

92 Clowse ME, Wallace DJ, Weisman M, et al. Predictors of preterm birth in patients with mild systemic lupus erythematosus. Ann Rheum Dis 2013;72:1536-9.

93 Lees C, Marlow N, Arabin B, et al. Perinatal morbidity and mortality in early-onset fetal growth restriction: cohort outcomes of the trial of randomized umbilical and fetal flow in Europe (TRUFFLE). Ultrasound Obstet Gynecol 2013;42:400-8. 
94 Lees CC, Marlow N, van Wassenaer-Leemhuis A, et al. 2 year neurodevelopmental and intermediate perinatal outcomes in infants with very preterm fetal growth restriction (TRUFFLE): a randomised trial. Lancet 2015;385:2162-72.

95 Alfirevic Z, Stampalija T, Gyte GM. Fetal and umbilical Doppler ultrasound in high-risk pregnancies. Cochrane Database Syst Rev 2013;11:CD007529.

96 Figueras F, Savchev S, Triunfo S, et al. An integrated model with classification criteria to predict small-for-gestational-age fetuses at risk of adverse perinatal outcome. Ultrasound Obstet Gynecol 2015;45:279-85.

97 Sovio U, White IR, Dacey A, et al. Screening for fetal growth restriction with universal third trimester ultrasonography in nulliparous women in the Pregnancy Outcome Prediction (POP) study: a prospective cohort study. Lancet 2015;386:2089-97.

98 Brito-Zerón P, Izmirly PM, Ramos-Casals M, et al. The clinical spectrum of autoimmune congenital heart block. Nat Rev Rheumatol 2015;11:301-12.

99 Saxena A, Izmirly PM, Mendez B, et al. Prevention and treatment in utero of autoimmune-associated congenital heart block. Cardiol Rev 2014;22:263-7.

100 Friedman DM, Kim MY, Copel JA, et al. Prospective evaluation of fetuses with autoimmune-associated congenital heart block followed in the PR Interval and Dexamethasone Evaluation (PRIDE) Study. Am J Cardiol 2009;103:1102-6.

101 Izmirly PM, Saxena A, Kim MY, et al. Maternal and fetal factors associated with mortality and morbidity in a multi-racial/ethnic registry of anti-SSA/Ro-associated cardiac neonatal lupus. Circulation 2011;124:1927-35.

102 Eliasson $\mathrm{H}$, Sonesson SE, Sharland G, et al. Isolated atrioventricular block in the fetus: a retrospective, multinational, multicenter study of 175 patients. Circulation 2011;124:1919-26.

103 Levesque K, Morel N, Maltret A, et al. Description of 214 cases of autoimmune congenital heart block: Results of the French neonatal lupus syndrome. Autoimmun Rev 2015;14:1154-60.

104 Izmirly PM, Saxena A, Sahl SK, et al. Assessment of fluorinated steroids to avert progression and mortality in anti-SSA/Ro-associated cardiac injury limited to the fetal conduction system. Ann Rheum Dis 2016;75:1161-5.

105 Tingström J, Hjelmstedt A, Welin Henriksson E, et al. Ro/SSA autoantibody-positive pregnancy: reactions to serial fetal Doppler echocardiographic surveillance. Lupus 2015;24:1540-5.

106 Derksen RH, Bruinse HW, de Groot PG, et al. Pregnancy in systemic lupus erythematosus: a prospective study. Lupus 1994;3:149-55.

107 Al Maimouni H, Gladman DD, Ibañez D, et al. Switching treatment between mycophenolate mofetil and azathioprine in lupus patients: indications and outcomes. Arthritis Care Res (Hoboken) 2014;66:1905-9.

108 Hussein MM, Mooij JM, Roujouleh H. Cyclosporine in the treatment of lupus nephritis including two patients treated during pregnancy. Clin Nephrol 1993:40:160-3.

109 Webster P, Wardle A, Bramham K, et al. Tacrolimus is an effective treatment for lupus nephritis in pregnancy. Lupus 2014;23:1192-6.

110 Moroni G, Quaglini S, Banfi G, et al. Pregnancy in lupus nephritis. Am J Kidney Dis 2002:40:713-20.

111 Götestam Skorpen C, Hoeltzenbein M, Tincani A, et al. The EULAR points to consider for use of antirheumatic drugs before pregnancy, and during pregnancy and lactation. Ann Rheum Dis 2016;75:795-810.

112 Abou-Nassar K, Karsh J, Giulivi A, et al. Successful prevention of thrombotic thrombocytopenic purpura (TTP) relapse using monthly prophylactic plasma exchanges throughout pregnancy in a patient with systemic lupus erythematosus and a prior history of refractory TTP and recurrent fetal loss. Transfus Apher Sci 2010;43:29-31.

113 Takeshita $Y$, Turumi $Y$, Touma $S$, et al. Successful delivery in a pregnant woman with lupus anticoagulant positive systemic lupus erythematosus treated with double filtration plasmapheresis. Ther Apher 2001;5:22-4.

114 Cavallasca JA, Laborde HA, Ruda-Vega H, et al. Maternal and fetal outcomes of 72 pregnancies in Argentine patients with systemic lupus erythematosus (SLE). Clin Rheumatol 2008;27:41-6.

115 Danve A, Perry L, Deodhar A. Use of belimumab throughout pregnancy to treat active systemic lupus erythematosus: a case report. Semin Arthritis Rheum 2014:44:195-7.

116 Østensen M, Förger F. How safe are anti-rheumatic drugs during pregnancy? Curr Opin Pharmacol 2013;13:470-5.

117 Flint J, Panchal S, Hurrell A, et al. BSR and BHPR guideline on prescribing drugs in pregnancy and breastfeeding-Part I: standard and biologic disease modifying anti-rheumatic drugs and corticosteroids. Rheumatology 2016 [epub ahead of print].

118 Flint J, Panchal S, Hurrell A, et al. BSR and BHPR guideline on prescribing drugs in pregnancy and breastfeeding-Part II: analgesics and other drugs used in rheumatology practice. Rheumatology 2016. [epub ahead of print].

119 Sciascia S, Hunt BJ, Talavera-Garcia E, et al. The impact of hydroxychloroquine treatment on pregnancy outcome in women with antiphospholipid antibodies. Am J Obstet Gynecol 2016;214:273.e1-8. [epub ahead of print].

120 Sciascia S, Branch DW, Levy RA, et al. The efficacy of hydroxychloroquine in altering pregnancy outcome in women with antiphospholipid antibodies. Evidence and clinical judgment. Thromb Haemost 2016;115:285-90.
121 Mekinian A, Lazzaroni MG, Kuzenko A, et al. The efficacy of hydroxychloroquine for obstetrical outcome in anti-phospholipid syndrome: data from a European multicenter retrospective study. Autoimmun Rev 2015;14:498-502.

122 Tunks RD, Clowse ME, Miller SG, et al. Maternal autoantibody levels in congenital heart block and potential prophylaxis with antiinflammatory agents. Am J Obstet Gynecol 2013;208:64.e1-7.

123 Roberge S, Villa P, Nicolaides K, et al. Early administration of low-dose aspirin for the prevention of preterm and term preeclampsia: a systematic review and meta-analysis. Fetal Diagn Ther 2012;31:141-6.

124 Wu CQ, Kustec VE, Brown RN, et al. The medical management of antiphospholipid syndrome in pregnancy: a meta-analysis. Obstet Gynecol 2014;123(Suppl 1):178s-9s.

125 Mak A, Cheung MW, Cheak AA, et al. Combination of heparin and aspirin is superior to aspirin alone in enhancing live births in patients with recurrent pregnancy loss and positive anti-phospholipid antibodies: a meta-analysis of randomized controlled trials and meta-regression. Rheumatology (Oxford) 2010;49:281-8.

126 Ziakas PD, Pavlou M, Voulgarelis M. Heparin treatment in antiphospholipid syndrome with recurrent pregnancy loss: a systematic review and meta-analysis. Obstet Gynecol 2010;115:1256-62.

127 de Jesús GR, Rodrigues $G$, de Jesús NR, et al. Pregnancy morbidity in antiphospholipid syndrome: what is the impact of treatment? Curr Rheumatol Rep 2014;16:403.

128 Ruffatti A, Gervasi MT, Favaro M, et al. Adjusted prophylactic doses of nadroparin plus low dose aspirin therapy in obstetric antiphospholipid syndrome. A prospective cohort management study. Clin Exp Rheumatol 2011;29:551-4.

129 Fouda UM, Sayed AM, Abdou AM, et al. Enoxaparin versus unfractionated heparin in the management of recurrent abortion secondary to antiphospholipid syndrome. Int J Gynaecol Obstet 2011;112:211-15.

130 Ruffatti A, Salvan E, Del Ross T, et al. Treatment strategies and pregnancy outcomes in antiphospholipid syndrome patients with thrombosis and triple antiphospholipid positivity. A European multicentre retrospective study. Thromb Haemost 2014;112:727-35

131 Bramham K, Thomas M, Nelson-Piercy C, et al. First-trimester low-dose prednisolone in refractory antiphospholipid antibody-related pregnancy loss. Blood 2011;117:6948-51.

132 Fischer-Betz R, Specker C, Brinks R, et al. Pregnancy outcome in patients with antiphospholipid syndrome after cerebral ischaemic events: an observational study. Lupus 2012;21:1183-9.

133 Le Thi Thuong D, Tieulié N, Costedoat N, et al. The HELLP syndrome in the antiphospholipid syndrome: retrospective study of 16 cases in 15 women. Ann Rheum Dis 2005;64:273-8.

134 Buyon JP, Petri MA, Kim MY, et al. The effect of combined estrogen and progesterone hormone replacement therapy on disease activity in systemic lupus erythematosus: a randomized trial. Ann Intern Med 2005;142 (Pt 1):953-62. http://dx.doi.org/10.7326/0003-4819-142-12_Part_ 1-200506210-00004

135 Sánchez-Guerrero J, Gonzalez-Perez M, Durand-Carbajal M, et al. Menopause hormonal therapy in women with systemic lupus erythematosus. Arthritis Rheum 2007;56:3070-9.

136 Cravioto MD, Durand-Carbajal M, Jiménez-Santana L, et al. Efficacy of estrogen plus progestin on menopausal symptoms in women with systemic lupus erythematosus: a randomized, double-blind, controlled trial. Arthritis Care Res (Hoboken) 2011:63:1654-63.

137 Hochman J, Urowitz MB, Ibañez D, et al. Hormone replacement therapy in women with systemic lupus erythematosus and risk of cardiovascular disease. Lupus 2009;18:313-17.

138 Fernandez M, Calvo-Alen J, Bertoli AM, et al. Systemic lupus erythematosus in a multiethnic US cohort (LUMINA L II): relationship between vascular events and the use of hormone replacement therapy in postmenopausal women. J Clin Rheumatol 2007; $13: 261-5$

139 Drake MT, Clarke BL, Lewiecki EM. The pathophysiology and treatment of osteoporosis. Clin Ther 2015;37:1837-50.

140 Sarri G, Davies M, Lumsden MA. Diagnosis and management of menopause: summary of NICE guidance. BMJ 2015;351:h5746.

141 National Collaborating Centre for Ws, Children's H. National Institute for Health and Clinical Excellence: Guidance. Menopause: Full Guideline. London: National Institute for Health and Care Excellence (UK) Copyright (c) 2015 National Collaborating Centre for Women's and Children's Health, 2015. https://www.nice. org.uk/guidance/ng23/evidence/appendices-ag-559549262

142 Bernatsky S, Ramsey-Goldman R, Foulkes WD, et al. Breast, ovarian, and endometrial malignancies in systemic lupus erythematosus: a meta-analysis. $\mathrm{Br} \mathrm{J}$ Cancer 2011;104:1478-81.

143 Bernatsky S, Ramsey-Goldman R, Labrecque J, et al. Cancer risk in systemic lupus: an updated international multi-centre cohort study. J Autoimmun 2013:42:130-5. 
144 Liu H, Ding Q, Yang K, et al. Meta-analysis of systemic lupus erythematosus and the risk of cervical neoplasia. Rheumatology (Oxford) 2011;50:343-8.

145 Santana IU, Gomes Ado N, Lyrio LD, et al. Systemic lupus erythematosus, human papillomavirus infection, cervical pre-malignant and malignant lesions: a systematic review. Clin Rheumatol 2011;30:665-72.

146 Zard E, Arnaud L, Mathian A, et al. Increased risk of high grade cervical squamous intraepithelial lesions in systemic lupus erythematosus: a meta-analysis of the literature. Autoimmun Rev 2014;13:730-5.

147 Dreyer L, Faurschou M, Mogensen M, et al. High incidence of potentially virus-induced malignancies in systemic lupus erythematosus: a long-term followup study in a Danish cohort. Arthritis Rheum 2011;63:3032-7.

148 Bateman H, Yazici Y, Leff L, et al. Increased cervical dysplasia in intravenous cyclophosphamide-treated patients with SLE: a preliminary study. Lupus 2000;9:542-4.

149 Bernatsky S, Ramsey-Goldman R, Gordon C, et al. Factors associated with abnormal Pap results in systemic lupus erythematosus. Rheumatology (Oxford) 2004;43:1386-9.

150 Ognenovski VM, Marder W, Somers EC, et al. Increased incidence of cervical intraepithelial neoplasia in women with systemic lupus erythematosus treated with intravenous cyclophosphamide. J Rheumatol 2004;31:1763-7.

151 Klumb EM, Araujo ML Jr, Jesus GR, et al. Is higher prevalence of cervical intraepithelial neoplasia in women with lupus due to immunosuppression? J Clin Rheumatol 2010;16:153-7.
152 Bernatsky SR, Cooper GS, Mill C, et al. Cancer screening in patients with systemic lupus erythematosus. J Rheumatol 2006;33:45-9.

153 Yazdany J, Tonner C, Trupin L, et al. Provision of preventive health care in systemic lupus erythematosus: data from a large observational cohort study. Arthritis Res Ther 2010;12:R84

154 Slade BA, Leidel L, Vellozzi C, et al. Postlicensure safety surveillance for quadrivalent human papillomavirus recombinant vaccine. JAMA 2009;302:750-7.

155 Soybilgic A, Onel KB, Utset $T$, et al. Safety and immunogenicity of the quadrivalent HPV vaccine in female Systemic Lupus Erythematosus patients aged 12 to 26 years. Pediatr Rheumatol Online J 2013;11:29.

156 Mok CC, Ho LY, Fong LS, et al. Immunogenicity and safety of a quadrivalent human papillomavirus vaccine in patients with systemic lupus erythematosus: a case-control study. Ann Rheum Dis 2013;72:659-64.

157 Soldevilla HF, Briones SF, Navarra SV. Systemic lupus erythematosus following HPV immunization or infection? Lupus 2012:21:158-61.

158 Gatto M, Agmon-Levin N, Soriano A, et al. Human papillomavirus vaccine and systemic lupus erythematosus. Clin Rheumatol 2013:32:1301-7.

159 Baker B, Eça Guimarães L, Tomljenovic L, et al. The safety of human papilloma virus-blockers and the risk of triggering autoimmune diseases. Expert Opin Drug Saf 2015;14:1387-94.

160 van Assen S, Agmon-Levin N, Elkayam 0, et al. EULAR recommendations for vaccination in adult patients with autoimmune inflammatory rheumatic diseases. Ann Rheum Dis 2011;70:414-22. 